\title{
Significant edges in the case of non-stationary Gaussian noise
}

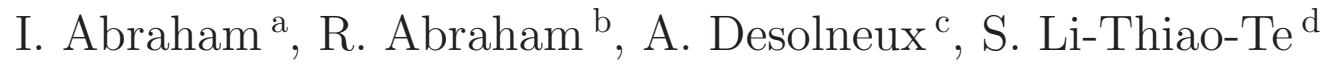 \\ ${ }^{a} C E A / D I F, 91680$ Bruyères le Chatel, France \\ ${ }^{\mathrm{b}}$ Laboratoire MAPMO, Fédération Denis Poisson, Université d'Orléans, \\ B.P. 6759, 45067 Orléans cedex 2, France \\ ${ }^{\mathrm{c}}$ Laboratoire MAP5, Université René Descartes, 45 rue des Saints-Pères, \\ 75270 Paris cedex 06, France \\ d Laboratoire CMLA, ENS Cachan, 61 avenue du Président Wilson, \\ 94235 Cachan cedex, France
}

\begin{abstract}
In this paper, we propose an edge detection technique based on some local smoothing of the image followed by a statistical hypothesis testing on the gradient. An edge point being defined as a zero-crossing of the Laplacian, it is said to be a significant edge point if the gradient at this point is larger than a threshold $s(\varepsilon)$ defined by: if the image $I$ is pure noise, then the probability of $\|\nabla I(x)\| \geq s(\varepsilon)$ conditionally on $\Delta I(x)=0$ is less than $\varepsilon$. In other words, a significant edge is an edge which has a very low probability to be there because of noise. We will show that the threshold $s(\varepsilon)$ can be explicitly computed in the case of a stationary Gaussian noise. In the images we are interested in, which are obtained by tomographic reconstruction from a radiograph, this method fails since the Gaussian noise is not stationary anymore. Nevertheless, we are still able to give the law of the gradient conditionally on the zero-crossing of the Laplacian, and thus compute the threshold $s(\varepsilon)$. We will end this paper with some experiments and compare the results with those obtained with other edge detection methods.
\end{abstract}

Key words: Edge detection, Significant edges, Inverse problem, Statistical hypothesis testing

Email addresses: isabelle.abraham@cea.fr (I. Abraham), romain.abraham@univ-orleans.fr (R. Abraham), 


\section{Introduction}

This work stems from analysis of physical experiments where a test object is submitted to a shock. More precisely, we surround the object with explosives and we monitor the shape of the hull as it first collapses onto itself and then expands. The shape of inners hole(s) at the very beginning of the re-expansion phase is of particular interest. We use radiography to determine those shapes. Standard tomography cannot be carried out because the inverse Radon transform requires many radiographs (taken from different angles) whereas only one radiograph can be acquired in the lapse of the expansion phase. That is why the hulls are supposed to be radially symmetric at the beginning of the experiment and during the explosion. In that case, a single radiograph is enough to perform the tomographic reconstruction (see [1] and [8], and Section 4.1).

Thus, from now on, we will only consider radially symmetric objects. To describe such an object, it is sufficient to provide the densities of the materials on a slice of the object that contains the symmetry axis. An example of studied object is given in Figure 1. The first step of the physical experiment is to performe a radiography of this object (see Figure 2(a)), then a tomographic reconstruction is computed (Figure 2(b)) and finally an edge detection is made (Figure 2(c)).

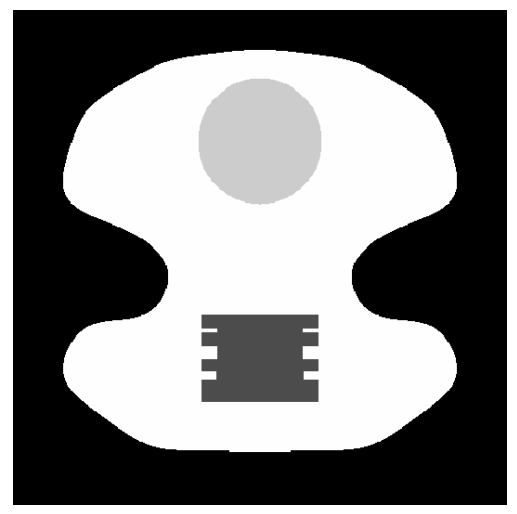

Fig. 1. Slice of a studied object.

agnes.desolneux@math-info.univ-paris5.fr (A. Desolneux), lithiaote@cmla.ens-cachan.fr (S. Li-Thiao-Te). 


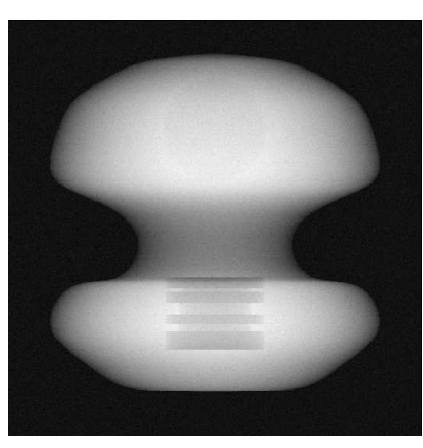

(a)

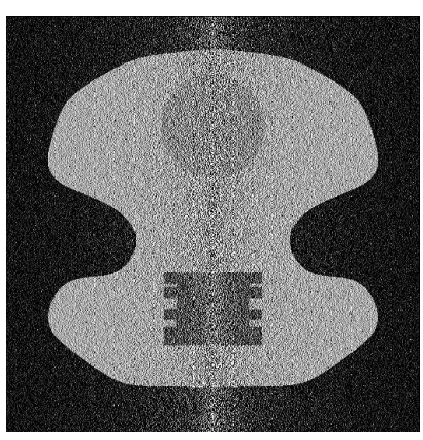

(b)

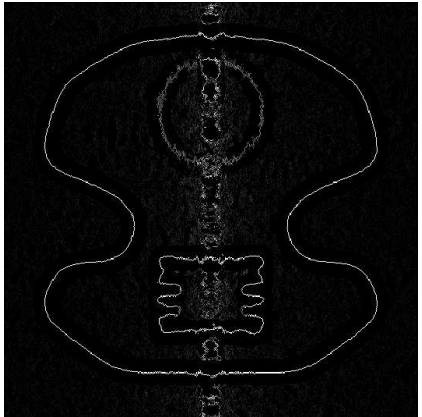

(c)

Fig. 2. (a) Radiograph of the object of Figure 1. (b) Tomographic reconstruction. (c) Edge detection on the tomography.

As we can see on Figure 2(c), many detected edges do not correspond to real features. This is due to the high level of noise. Our goal is to perform an automated selection of the "true" edges. For that purpose, the edge detector will not be changed but we will compute also other significant features that will allow us to select the "true" edges. Let us stress that our goal is not to develop a new edge detector but to give a method of selection of edges from the map given by an edge detector. We focus here on an edge detector which is certainly not optimal but where the computations are easy. However, we think that our method can be applied to other edge detectors such as the Canny detector [3]. In his paper, Canny points out the fact that "it is very difficult to set a threshold so that there is small probability of marking noise edges while retaining high sensitivity". And the solution he proposes to this problem is the use of hysteresis. Here, we will use the zero-crossings of the Laplacian to define edge points. We will see that in this case, we can compute a threshold so that there is a small probability of selecting edges due to noise.

The ideas used here mainly come from previous work of Desolneux, Moisan and Morel [6]. Informally speaking, they define the notion of significant edges by computing the probability that some edge-related events appear in an image of pure noise. When this probability is small enough, the edge is probably a feature of the image and not due to noise. Unfortunately, that method assumes that the noise is stationary (i.e. the law of the noise does not depend on the location in the image) which, as is easily seen on Figure 2(b), is not the case in our study because of the tomographic inversion (see Section 4.5.1 for some examples of results obtained with their method). Moreover, their study is quite general and, apart from the stationarity, no assumption is made on the noise.

In our case, as we deal with specific images, the noise is well-known and some statistical models can be used. Indeed, we may suppose that the noise on the radiograph (Figure 2(a)) is a Gaussian white noise with mean zero and with a variance that can easily be estimated. After tomographic inversion, we still 
obtain Gaussian noise because that operation is linear, but the noise process is correlated and non-stationary.

The edge detector we will use here consists in estimating the Laplacian at each point, and edge points are then defined as the zero-crossings of the Laplacian. As already said, we will compute additional features that discriminate the significant edges. The easiest feature to compute is a kind of contrast measurement $C$ based on a gradient estimate. To be more precise, we consider an image $I$ of pure noise (that is a realization of our model of noise after tomographic reconstruction), estimate the gradient and the Laplacian of $I$ at a point $(u, v)$ (with an abuse of notation, we will denote by $\nabla I(u, v)$ and $\Delta I(u, v)$ these estimates and by $C(u, v)$ the contrast value) and we compute, for a fixed $\varepsilon>0$, the smallest value $s(\varepsilon)$ such that

$$
\mathbb{P}(C(u, v) \geq s(\varepsilon) \mid \Delta I(u, v)=0) \leq \varepsilon
$$

where $\mathbb{P}(B \mid A)$ denotes the conditional probability of $B$ given the event $A$.

Then, we perform an edge detection on the studied image $f$ (where we also estimate $\nabla f$ and $\Delta f$ by the same method) and we keep the points $(u, v)$ of the studied image $f$ that satisfy

- $\Delta f(u, v)=0$ (an edge is present at point $(u, v))$.

- $C(u, v) \geq s(\varepsilon)$ (this edge is significant).

From a statistical point of view, this consists in performing an hypothesis test. We consider a point $(u, v)$ where an edge takes place $(\Delta(u, v)=0)$ and we test the null hypothesis "the zero-crossing of the Laplacian is due to the noise". The level of the test $\varepsilon$ is arbitrarily chosen and related to the number of false detections allowed. It will be set to $\varepsilon=10^{-5}$ hereafter (this corresponds to 2.6 errors on average in a $512 \times 512$ image). Let us mention that the threshold value $s(\varepsilon)$ varies slowly with respect to $\varepsilon$. For instance, in the case of a white noise (see Section 3), the threshold value can be computed explicitly and is proportional to $\sqrt{-\ln \varepsilon}$. This implies that when we increase the specificity (less false detections), the threshold increases slowly and the method retains much of its sensitivity. When the null hypothesis is rejected, the edge is retained as it comes from a "true" feature of the image, whereas when the null hypothesis is accepted, the zero-crossing of the Laplacian may come from the noise and the edge is not meaningful.

Let us mention at this point that such statistical approaches have already been used for edge detection in [18], [16] or [13]. They usually employ estimates of the gradient based on finite differences which fail in our case because these are not robust enough to high levels of noise. Moreover, the noise is in most cases stationary. Let us also cite [4] where the authors have modified the method of 
[6] to take into account the non-stationarity of some images, by a local noise estimate. Their work is still general and does not make any assumption on the noise structure. As we deal with specific experiments, the noise is always the same and well-known and we can take proper advantage of this knowledge. The problem of finding an optimal threshold is also a key point for image thresholding methods [17] and particularly for the binarization of document images [19]. In some cases, the thresholding is not stationnary: this happens for instance in images with non-uniform illumination. Our framework here is different since we deal with a well-known noise, and the question we address is: given an edge detection method, what is the probability that an edge point is due to noise?

The paper is organized as follows: in Section 2, we present the edge detector based on the estimates of the gradient and of the Laplacian. Then, in Section 3, our method is presented in the case of a Gaussian white noise. Of course, this does not correspond to our case but the computations are easier and demonstrate the performance of this framework. In Section 4, we will first describe the tomographic inversion and the operators involved, and then describe the noise model we have to deal with. We will then apply the significant edges detection method in the framework of this non-stationary noise. We will end the section with some experiments and comparisons with other methods.

\section{Estimating the Gradient and the Laplacian}

In this section, we introduce the method for edge detection. We consider that the image is a real-valued function $(u, v) \mapsto f(u, v)$ of two continuous real parameters $u$ and $v$. Then, we say that there exists an edge at point $(u, v)$ if the Laplacian of $f$ is zero at this point. Moreover, the computation of the contrast function $C$ will be based on the gradient of $f$ (see the end of this section for the choice of this function). As the images are very noisy, these derivatives cannot be estimated by usual finite differences. The method used here, sometimes known as Savitsky-Golay smoothing, consists in locally approximating the image by a polynomial. The derivatives of the polynomial are then identified with those of the image.

\subsection{An optimization problem}

Let $(u, v)$ denote the point where we want to compute the first and second order derivatives of the image $f$. We choose 2 parameters : $d$ which is the maximum degree of the approximating polynomial and $r$ which is the radius of the ball on which we perform the approximation. We denote by $B_{r}(u, v)$ 
the ball of radius $r$ centered at point $(u, v)$. We will simply write $B_{r}$ when the center of the ball is the origin $(0,0)$ of $\mathbb{R}^{2}$. We are then looking for a polynomial $P$ of degree less that $d$ such that

$$
E(P)=\int_{B_{r}}(f(u+x, v+y)-P(x, y))^{2} d x d y
$$

is minimal among all polynomials of degree less than $d$. In other words, we are looking for the best approximation of $f$ by a polynomial of degree less than $d$ on the ball $B_{r}(u, v)$ in the sense of the $L^{2}$-norm.

This is an optimization problem where the unknowns are the coefficients of the polynomial. As the problem is convex, there is a unique solution (given by the orthogonal projection of $f$ on the space of polynomials of degree less than $d$ ) which is easily computed by solving the set of equations

$$
\frac{\partial E}{\partial a_{i}}=0
$$

where the $a_{i}$ 's denote the coefficients of the polynomial.

Role of the ball radius. Two parameters are arbitrary chosen in this method. The first one is the ball radius $r$. The larger $r$ is, the more effective the smoothing is. The influence of the noise is therefore attenuated with a large $r$ but the location of the edge is then less precise. We must consequently make a balance between noise smoothing and edge detection accuracy. For instance, if we have a small level of noise or if the edges are very complicated (with high curvature), we must choose a small value for $r$.

Role of the polynomial degree. The second parameter is the polynomial degree. Here again a large value of $d$ gives a better approximation but does not smooth the noise enough. In fact, as we are, in a first step, interested in the points where the Laplacian is zero, it appears that a second-order polynomial is enough. Of course, the estimate of the first order derivatives with a polynomial of degree 2 is not very good and highly depends on the size of the window $B_{r}$. But we will see that this drawback can be useful for the choice of a contrast function.

Choice of the $L^{2}$-norm. Approximating a function in a $L^{2}$-sense, although quite usual, is not always accurate as some oscillations may appear or local bad approximations are allowed (this usually occurs near the boundary of the domain). However, as we will only look for polynomials of degree 2, the oscillations do not appear and the $L^{2}$-norm gives a good enough approximation together with easy computations.

In what follows, the approximation is made with a polynomial of degree $d=2$, and the first and second order derivatives of the image are identified with those of the approximating polynomial. 


\subsection{Computations with a second order polynomial}

Let us first introduce some notations. In the following, we will set

$$
\forall i, j \in \mathbb{N}, \quad b_{i j}(r)=\int_{B_{r}} x^{i} y^{j} d x d y
$$

As the ball $B_{r}$ is symmetric, we have that $b_{i j}(r)=0$ if $i$ or $j$ is odd and that $b_{i j}(r)=b_{j i}(r)$ for all $i, j$. In order to have simple expressions, we will also set:

$$
b(r)=b_{20}(r), \quad \alpha(r)=-\frac{2 b_{20}(r)}{b_{00}(r)} \text { and } \beta(r)=\frac{1}{2}\left(b_{40}(r)+b_{22}(r)-\frac{2 b_{20}^{2}(r)}{b_{00}(r)}\right)
$$

Lemma 1 The gradient and the Laplacian of the polynomial $P$ of degree 2 which is the best approximation of $f$ on the ball $B_{r}(u, v)$ for the $L^{2}$-norm, being respectively denoted by $\nabla_{r} f(u, v)=\left(\frac{\partial_{r} f}{\partial x}(u, v), \frac{\partial_{r} f}{\partial y}(u, v)\right)$ and $\Delta_{r} f(u, v)$, are given by:

$$
\begin{aligned}
& \frac{\partial_{r} f}{\partial x}(u, v)=\frac{1}{b(r)} \int_{B_{r}} x f(u+x, v+y) d x d y \\
& \frac{\partial_{r} f}{\partial y}(u, v)=\frac{1}{b(r)} \int_{B_{r}} y f(u+x, v+y) d x d y \\
& \Delta_{r} f(u, v)=\frac{1}{\beta(r)} \int_{B_{r}} f(u+x, v+y)\left(\alpha(r)+x^{2}+y^{2}\right) d x d y
\end{aligned}
$$

Proof :

We consider a polynomial of degree 2 which we write

$$
P(x, y)=a_{x x} x^{2}+a_{y y} y^{2}+a_{x y} x y+a_{x} x+a_{y} y+a_{0} .
$$

The equations obtained by writing $\nabla E(P)=0$, where $E(P)$ is given by Equation (2), are:

$$
\left\{\begin{aligned}
b_{40}(r) a_{x x}+b_{22}(r) a_{y y}+b_{20}(r) a_{0} & =\int_{B_{r}} x^{2} f(u+x, v+y) d x d y \\
b_{22}(r) a_{x x}+b_{40}(r) a_{y y}+b_{20}(r) a_{0} & =\int_{B_{r}} y^{2} f(u+x, v+y) d x d y \\
b_{22}(r) a_{x y} & =\int_{B_{r}} x y f(u+x, y+v) d x d y \\
& =\int_{B_{r}} x f(u+x, y+v) d x d y \\
b_{20}(r) a_{x} & =\int_{B_{r}} y f(u+x, v+y) d x d y \\
b_{20}(r) a_{y} & =\int_{B_{r}} f(u+x, v+y) d x d y
\end{aligned}\right.
$$


We then obtain the following estimates for the derivatives:

$$
\begin{aligned}
\frac{\partial P}{\partial x}(0,0) & =a_{x}=\frac{1}{b_{20}(r)} \int_{B_{r}} x f(u+x, v+y) d x d y \\
\frac{\partial P}{\partial y}(0,0) & =a_{y}=\frac{1}{b_{20}(r)} \int_{B_{r}} y f(u+x, v+y) d x d y \\
\Delta P(0,0) & =2\left(a_{x x}+a_{y y}\right) \\
& =\frac{2}{b_{40}+b_{22}-\frac{2 b_{20}^{2}}{b_{00}}} \int_{B_{r}} f(u+x, v+y)\left(-\frac{2 b_{20}}{b_{00}}+x^{2}+y^{2}\right) d x d y .
\end{aligned}
$$

\subsection{Choice of the contrast function}

We would like to use a contrast function based on the estimates of the first and second derivatives of the image $f$ obtained in the previous section.

The simplest contrast function we can choose is the norm of the gradient:

$$
C_{1}(u, v)=\left\|\nabla_{r} f(u, v)\right\| .
$$

Indeed, the value of this norm tells how sharp the edge is. This contrast function performs reasonably well and will be used when the images we deal with are piecewise constant.

However, in many cases, the objects we handle are not homogeneous and their images contain some slopes (see Figure 3 ). In this case, the gradient norm is not a good contrast function. Indeed, let us consider an image with a constant slope with some noise (see Figure 4). We would like to say that no edge is significant in that case. However, the value of the gradient norm (which will be close to the value of the slope) will always be greater than the threshold value $s$ when the noise level is small.

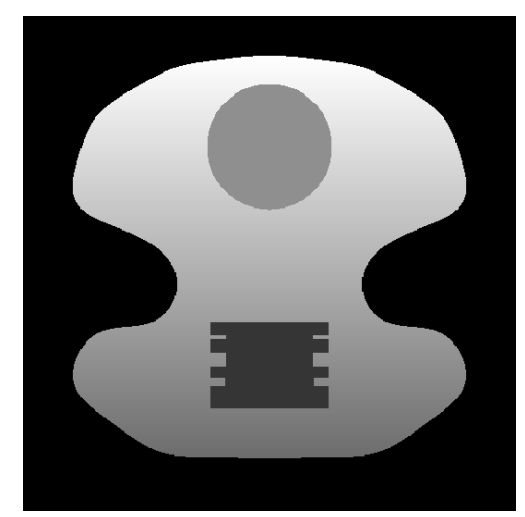

Fig. 3. Object with an inhomogeneous material 
In the latter case, we take advantage of the dependence of the first order derivatives estimates with respect to the ball radius. Indeed, the estimates of the gradient in the case of the constant slope in Figure 4 will not depend on the size of the window (see Figure 4) whereas, when an edge (a discontinuity) occurs, the estimates do depend on that radius (see Figure 5). So, we can use as a contrast function the function

$$
C_{2}(u, v)=\left\|\nabla_{r_{1}} f(u, v)-\nabla_{r_{2}} f(u, v)\right\|
$$

where $r_{1}<r_{2}$ and $\nabla_{r} f$ denotes the value of the gradient estimate with a ball of radius $r$.

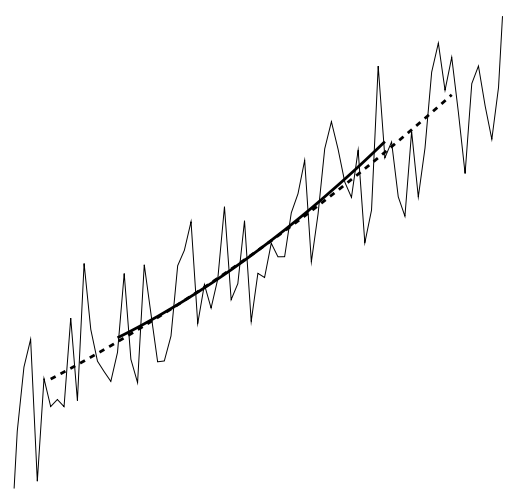

Fig. 4. A noisy constant slope: the gradient of the approximating polynomial does not depend on the radius $r$.

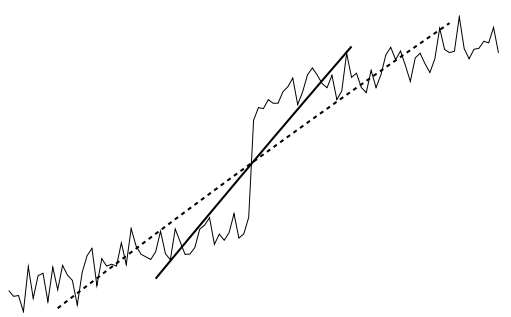

Fig. 5. An edge on a noisy slope: the approximating polynomials with two different values of the radius $r$.

\section{Significant edges in the case of a Gaussian white noise}

\subsection{White noise and Wiener integral}

We recall here the definition and the main properties of a white noise in a continuous setting and of the Wiener integral. We refer to [20], [10] or [11] for more on white noise and the Wiener integral. 
Definition 1 A Gaussian white noise on $\mathbb{R}^{2}$ of variance $\sigma^{2}$ is a random function $W$ defined on the Borel sets $A$ of $\mathbb{R}^{2}$ of finite Lebesgue measure (denoted by $|A|)$ such that

- $W(A)$ is a Gaussian random variable (r.v.) with mean 0 and variance $\sigma^{2}|A|$,

- If $A_{1} \cap A_{2}=\emptyset$, the r.v. $W\left(A_{1}\right)$ and $W\left(A_{2}\right)$ are independent and

$$
W\left(A_{1} \cup A_{2}\right)=W\left(A_{1}\right)+W\left(A_{2}\right) .
$$

Such a function $W$ exists but is not a true measure since the two-parameters process

$$
B(s, t):=W((0, s] \times(0, t])
$$

(with the convention that $(0, x]$ is $[x, 0)$ when $x$ is negative) is of unbounded total variation. This process is usually called the Brownian sheet.

Nevertheless we can define the so-called Wiener integral $\int f d W$ for every function $f$ in $L^{2}\left(\mathbb{R}^{2}\right)$. We can also define the derivatives of the Brownian sheet in the sense of Schwartz distributions (although the Brownian sheet is nowhere differentiable). Thus, we define

$$
\dot{B}(s, t)=\frac{\partial^{2} B(s, t)}{\partial s \partial t}
$$

and we have

$$
\int f d W=\int_{\mathbb{R}^{2}} f(u, v) \dot{B}(u, v) d u d v \quad \text { a.s. }
$$

for every function $f$ in the Schwartz space.

With a slight abuse of notations, we call $\dot{B}$ a Gaussian white noise and we always denote by $\int_{\mathbb{R}^{2}} f(u, v) \dot{B}(u, v) d u d v$ the Wiener integral with respect to this white noise, for every function $f \in L^{2}$. The main properties of this integral are

- For every $f$, the r.v. $\int_{\mathbb{R}^{2}} f(u, v) \dot{B}(u, v) d u d v$ is a Gaussian r.v. with mean 0 and variance $\sigma^{2} \int_{\mathbb{R}^{2}} f(u, v)^{2} d u d v$.

- For every $f, g$, the random vector

$$
\left(\int_{\mathbb{R}^{2}} f(u, v) \dot{B}(u, v) d u d v, \int_{\mathbb{R}^{2}} g(u, v) \dot{B}(u, v) d u d v\right)
$$

is Gaussian with covariance

$$
\sigma^{2} \int_{\mathbb{R}^{2}} f(u, v) g(u, v) d u d v
$$

We will use these properties to compute the laws of $\nabla I$ and $\Delta I$. 


\subsection{Laws of the gradient and of the Laplacian}

We suppose here that our noise is a Gaussian white noise, of variance $\sigma^{2}$. As we have already said, this case is not the one we are interested in and our method is probably out-performed by other standard methods in that case. The goal of this section is to present our method in a simple case where the computations are easy and can be carried out in a continuous setting. We will only focus here on the case of piecewise constant objects and therefore we will use the contrast function $C_{1}$.

Lemma 2 If the image $I$ is a Gaussian white noise of variance $\sigma^{2}$, then

$$
\left(\frac{\partial_{r} I}{\partial x}, \frac{\partial_{r} I}{\partial y}, \Delta_{r} I\right)
$$

is a Gaussian vector with mean zero and covariance matrix

$$
\left(\begin{array}{ccc}
\frac{\sigma^{2}}{b(r)} & 0 & 0 \\
0 & \frac{\sigma^{2}}{b(r)} & 0 \\
0 & 0 & V(r, \sigma)
\end{array}\right) \text {, where } V(r, \sigma)=\frac{\sigma^{2}}{\beta^{2}(r)} \int_{B_{r}}\left(\alpha(r)+x^{2}+y^{2}\right)^{2} d x d y
$$

Proof: We compute the laws of the approximate derivatives of $I$ when $I=\dot{B}$. We recall that these derivatives are given by

$$
\begin{aligned}
& \frac{\partial_{r} I}{\partial x}(u, v)=\frac{1}{b(r)} \int_{B_{r}} x \dot{B}(u+x, v+y) d x d y \\
& \frac{\partial_{r} I}{\partial y}(u, v)=\frac{1}{b(r)} \int_{B_{r}} y \dot{B}(u+x, v+y) d x d y \\
& \Delta_{r} I(u, v)=\frac{1}{\beta(r)} \int_{B_{r}} \dot{B}(u+x, v+y)\left(\alpha(r)+x^{2}+y^{2}\right) d x d y
\end{aligned}
$$

Because of the stationarity of $\dot{B}$, they have the same law as

$$
\begin{aligned}
\frac{\partial_{r} I}{\partial x}(0,0) & =\frac{1}{b(r)} \int_{B_{r}} x \dot{B}(x, y) d x d y \\
\frac{\partial_{r} I}{\partial y}(0,0) & =\frac{1}{b(r)} \int_{B_{r}} y \dot{B}(x, y) d x d y \\
\Delta_{r} I(0,0) & =\frac{1}{\beta(r)} \int_{B_{r}} \dot{B}(x, y)\left(\alpha(r)+x^{2}+y^{2}\right) d x d y
\end{aligned}
$$


As we deal with Wiener integrals, we deduce that the vector

$$
\left(\frac{\partial_{r} I}{\partial x}, \frac{\partial_{r} I}{\partial y}, \Delta_{r} I\right)
$$

is a Gaussian vector with mean zero.

To compute its covariance matrix, let us recall that, if $X$ and $Y$ are random variables defined by

$$
\begin{aligned}
& X=\int_{B_{r}} h_{1}(x, y) \dot{B}(x, y) d x d y \\
& Y=\int_{B_{r}} h_{2}(x, y) \dot{B}(x, y) d x d y
\end{aligned}
$$

then we have

$$
\operatorname{Cov}(X, Y)=\sigma^{2} \int_{B_{r}} h_{1}(x, y) h_{2}(x, y) d x d y
$$

Consequently, we have for instance:

$$
\operatorname{Cov}\left(\frac{\partial_{r} I}{\partial x}, \frac{\partial_{r} I}{\partial y}\right)=\frac{\sigma^{2}}{b^{2}(r)} \int_{B_{r}} x y d x d y=0 .
$$

By some analogous calculations, we finally get the following covariance matrix for our Gaussian vector:

$$
\left(\begin{array}{ccc}
\frac{\sigma^{2}}{b(r)} & 0 & 0 \\
0 & \frac{\sigma^{2}}{b(r)} & 0 \\
0 & 0 & V(r, \sigma)
\end{array}\right)
$$

where

$$
V(r, \sigma)=\frac{\sigma^{2}}{\beta^{2}(r)} \int_{B_{r}}\left(\alpha(r)+x^{2}+y^{2}\right)^{2} d x d y
$$

Let us recall that two uncorrelated components of a Gaussian vector are independent random variables (which is not the case in general). Therefore we immediately have the following properties:

- The random variable $\left\|\nabla_{r} I\right\|^{2}$ is the sum of two squared independent Gaussian random variables which have the same variance. It is therefore distributed as a $\chi^{2}$-law. More precisely, its law is

$$
\frac{\sigma^{2}}{b(r)} \chi^{2}(2)
$$

where $\chi^{2}(2)$ denotes a $\chi^{2}$-law with two degrees of freedom. 
- The random variable $\Delta_{r} I$ is a Gaussian random variable with mean zero and variance $V(r, \sigma)$.

- The random variables $\left\|\nabla_{r} I\right\|^{2}$ and $\Delta_{r} I$ are independent.

\subsection{Computation of the threshold}

Proposition 1 Let I be a Gaussian white noise and let $s(\varepsilon)$ be the threshold value such that

$$
\mathbb{P}\left(\left\|\nabla_{r} I\right\| \geq s(\varepsilon) \mid \Delta_{r} I=0\right) \leq \varepsilon
$$

Then

$$
s(\varepsilon)=\sqrt{-\frac{2 \sigma^{2}}{b(r)} \ln \varepsilon} .
$$

Proof: To begin with, as the random variables $\left\|\nabla_{r} I\right\|^{2}$ and $\Delta_{r} I$ are independent, we can forget the conditioning and only compute

$$
\mathbb{P}\left(\left\|\nabla_{r} I\right\| \geq s(\varepsilon)\right)=\mathbb{P}\left(\left\|\nabla_{r} I\right\|^{2} \geq s(\varepsilon)^{2}\right) .
$$

As a consequence of Lemma 2, we have that the law of $\left\|\nabla_{r} I\right\|^{2}$ is $\frac{\sigma^{2}}{b(r)} \chi^{2}(2)$. Now, since the density of a $\chi^{2}(2)$ law is the one of a $\Gamma\left(\frac{1}{2}, 1\right)$ law, we have that the law of $\left\|\nabla_{r} I\right\|^{2}$ is given by

$$
\mathbb{P}\left(\left\|\nabla_{r} I\right\|^{2} \geq s^{2}\right)=\int_{\frac{b(r)}{\sigma^{2}} s^{2}}^{+\infty} \frac{1}{2} e^{-\frac{t}{2}} d t=\exp \left(-\frac{b(r) s^{2}}{2 \sigma^{2}}\right) .
$$

This finally leads to the announced threshold value $s(\varepsilon)$.

\subsection{Experiments}

We consider the piecewise constant object of Figure 1 with some additive Gaussian white noise. The densities of the different materials of this object are:

- 1 for the outer material,

- 0.8 for the material inside the circle,

- 0.3 for the other inner material.

The standard deviation of the Gaussian noise is $\sigma=0.2$ in the experiments of Figure 6 and is $\sigma=0.4$ in the experiments of Figure 7. Both images have the same size $512 \times 512$ pixels. The experiments have been carried out with a 
ball of radius $r=12$ pixels.

The different images of Figures 6 and 7 are respectively:

- (a) The noisy image.

- (b) The zero-crossings of the Laplacian with the contrast function $C_{1}$ visualized in grey-level (the white color corresponds to high value for the contrast function $C_{1}$ ).

- (c) The extracted significant edges $\left(\varepsilon=10^{-5}\right)$.

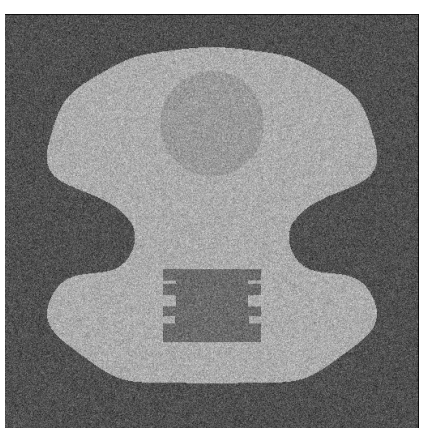

(a)

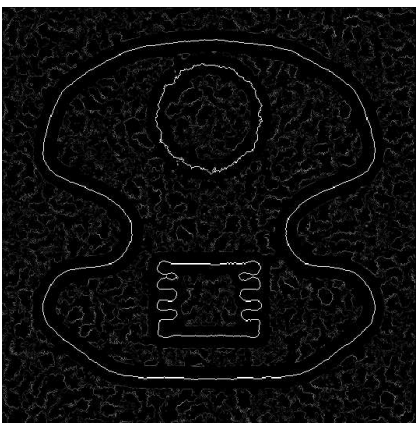

(b)

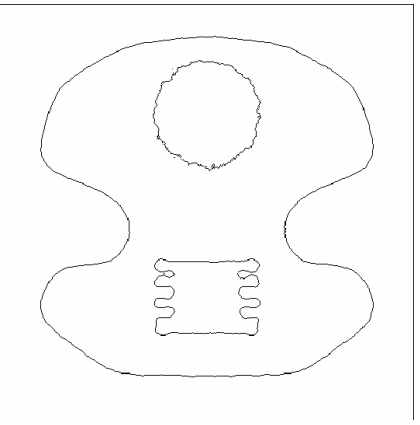

(c)

Fig. 6. (a) The noisy image $(\sigma=0.2)$. (b) The zero-crossings of the Laplacian with the contrast function $C_{1}$ visualized in grey-level. (c) The extracted significant edges $\left(\varepsilon=10^{-5}\right)$.

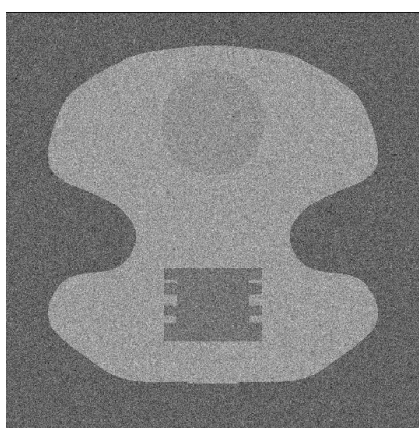

(a)

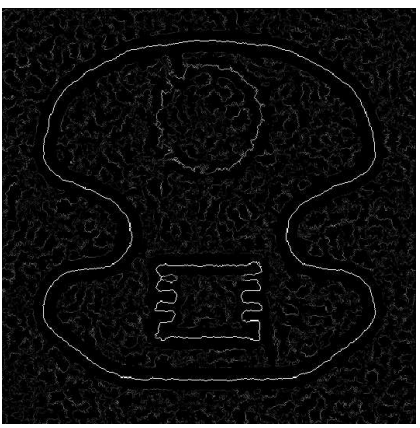

(b)

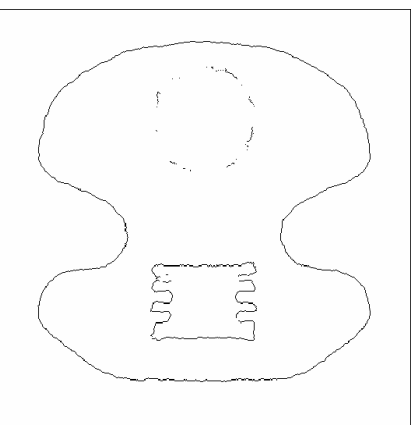

(c)

Fig. 7. (a) The noisy image $(\sigma=0.4)$. (b) The zero-crossings of the Laplacian with the contrast function $C_{1}$ visualized in grey-level. (c) The extracted significant edges $\left(\varepsilon=10^{-5}\right)$.

In the case of a signal-to-noise ratio large enough (Figure 6), all the edges are well detected and the "false" edges are removed. However, the edges which have a high curvature are smoothed by our algorithm. This drawback is all the more important when the ball radius $r$ is large (the influence of the value of this radius will be studied in the experiments of the next section). 
When the noise level is rather large (Figure 7), some edges of the image cannot be extracted from the noise (this happens when the contrast associated to this edge is close to the noise level).

\section{Significant edges in the case of Gaussian white noise on a radio- graph}

\subsection{Tomography}

Let us now turn to the more realistic case we are interested in. As we mentioned in the introduction, we first make a radiography of an object. Tomography is the inverse problem associated with reconstructing the initial object from its radiograph. This is now a well-known problem as it is the key tool in medical scanner imagery (or other medical imaging systems).

To begin with, let us describe what radiography is from a mathematical point of view. The studied object is exposed to $X$-rays that traverse it and some of the $X$-photons are absorbed. In the output, we observe the quantity of $X$ photons that have not been absorbed by the material, and we thus measure in some sense the "mass" of material the ray went through. More precisely, if the object is described by its density $\mu$ (which is a function of the space coordinates), what can be measured at any point of the receptor is

$$
\int_{\text {ray }} \mu d \ell
$$

where "ray" means the straight line that goes from the source to the studied point of the receptor (we suppose that the X-ray source is just a point, which implies that the previous line is unique).

We also assume that the X-ray source is far away from the object so that the rays are assumed to be all parallel. Then, to reconstruct an object from its radiographs, we must rotate the object and take a radiograph for every angle $\theta \in[0, \pi)$. This leads to the so-called Radon transform of the object, which is known to be invertible. This is the principle of the medical scanner.

In our case, as the object is radially symmetric, if we rotate around its symmetry axis, all the radiographs are exactly the same. Consequently, a single radiograph of such an object is enough to perform the tomographic reconstruction. Indeed, if $f(x, y)$ denotes the density along a slice that contains the symmetry axis (see Figures 1 and 8), then a radiograph of this object is given 
by

$$
g(u, v)=2 \int_{|u|}^{+\infty} f(x, v) \frac{x}{\sqrt{x^{2}-u^{2}}} d x .
$$

This is a linear transform and we will denote it hereafter by

$$
g=H f
$$

As we already said, this linear operator $H$ is invertible and we in fact know explicitly its inverse on the space of continuously differentiable functions $g$ :

$$
f(x, y)=\left(H^{-1} g\right)(x, y)=-\frac{1}{\pi} \int_{x}^{+\infty} \frac{1}{\sqrt{u^{2}-x^{2}}} \frac{\partial g}{\partial u}(u, y) d u .
$$

(The proof of this formula and more details about the operators $H$ and $H^{-1}$ can be found in the book of R.N. Bracewell [2] pp. 244-250).

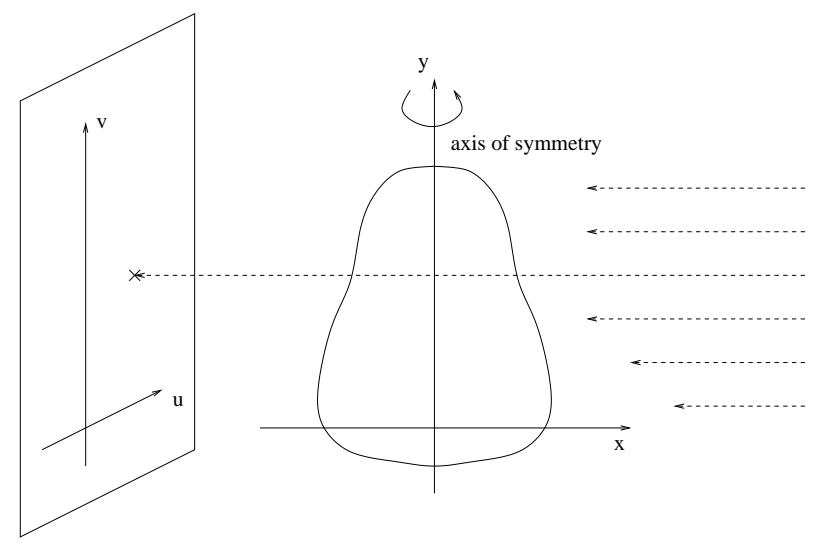

Fig. 8. Radiography of a radially symmetric object.

Our assumption on the noise is that it is an additive Gaussian white noise on the radiograph (i.e. on $g$ ). But what we want is to study the object given by $f$. So we must transform the white noise by the operator $H^{-1}$. Unfortunately, because of the singularity of the integral at $x=0$, we cannot apply the operator $H^{-1}$ to a white noise $\dot{B}$, even in a $L^{2}$-sense. Therefore, we will work in a discrete framework: the images $f$ and $g$ are naturally discretized (as they are numerical images). This leads to a discretization of the operator $H$, which we will still denote by $H$ and which now may be viewed as a matrix. The discretization is made in such a way that the symmetry axis $(x=0)$ is settled between two pixels so that the previous singularity does not appear. This matrix is then invertible and we denote by $\mathrm{H}^{-1}$ its inverse which we can make now operate on a discrete Gaussian white noise.

To give an idea of the ill-conditionedness of the matrix $H$, we can compute its condition number. For an image of size $512 \times 512$, we find that this condition number is 377 , which shows how $H^{-1}$ amplifies noise. 


\subsection{Law of the noise on the tomographic reconstruction}

Let us consider a field $\eta=\left(\eta_{i, j}\right)_{1 \leq i \leq p, 1 \leq j \leq n}$ of independent identically distributed (i.i.d.) random Gaussian variables with mean 0 and variance $\sigma^{2}$. Let us define $I=\left(I_{i, j}\right)$ the random field obtained after tomographic reconstruction, i.e. after making $H^{-1}$ operate on $\eta=\left(\eta_{i, j}\right)$. In fact, as the X-rays are supposed to be parallel, the reconstruction can be made line by line independently and therefore, if we consider the row vectors

$$
\vec{\eta}_{i}=\left(\eta_{i, 1}, \ldots, \eta_{i, n}\right) \text { and } \vec{I}_{i}=\left(I_{i, 1}, \ldots, I_{i, n}\right)
$$

then, if we denote $M=\left(H^{t}\right)^{-1}$ (where $H^{t}$ is the transpose of $H$ ), we have

$$
\vec{I}_{i}=\vec{\eta}_{i} M
$$

(Notice that $M$ is independent of $i$ and that its size is $n \times n$ ).

Consequently, the law of $I$ is characterized by the following properties:

- $I=\left(I_{i, j}\right)$ is a Gaussian random field.

- For $i \neq k, \vec{I}_{i}$ and $\vec{I}_{k}$ are independent.

- For each $i$, the vector $\vec{I}_{i}$ is a Gaussian vector of mean 0 and covariance matrix

$$
\Gamma=\sigma^{2} M^{t} M
$$

where $M^{t}$ denotes the transpose of $M$.

\subsection{Laws of the gradient and of the Laplacian}

The expressions obtained in Section 2 for the gradient and for the Laplacian of an image in a continuous setting are easily translated in the discrete framework we now deal with. Indeed, we have

$$
\begin{aligned}
& \frac{\partial_{r} I}{\partial x}(u, v)=\frac{1}{b(r)} \sum_{(i, j) \in B_{r}} j I_{u+i, v+j} \\
& \frac{\partial_{r} I}{\partial y}(u, v)=\frac{1}{b(r)} \sum_{(i, j) \in B_{r}} i I_{u+i, v+j} \\
& \Delta_{r} I(u, v)=\frac{1}{\beta(r)} \sum_{(i, j) \in B_{r}}\left(\alpha(r)+i^{2}+j^{2}\right) I_{u+i, v+j}
\end{aligned}
$$

where $B_{r}$ now denotes the discrete ball of radius $r$ i.e.

$$
B_{r}=\left\{(i, j), i^{2}+j^{2} \leq r^{2}\right\}
$$


and where the constants $\alpha(r), \beta(r), b(r), \ldots$ are the discrete analogues of the constants in Section 2.

With these estimates, the contrast functions $C_{1}$ and $C_{2}$ are easily comptuted. They are both of the form

$$
C(u, v)=\sqrt{C_{x}^{2}(u, v)+C_{y}^{2}(u, v)}
$$

with

$$
C_{x}(u, v)=\sum_{i, j} j c_{i j} I_{u+i, v+j} \quad \text { and } \quad C_{y}(u, v)=\sum_{i, j} i c_{i j} I_{u+i, v+j}
$$

where the coefficients $c_{i j}$ are given by:

(1) In the case of the contrast function $C_{1}$,

$$
c_{i j}=\frac{1}{b(r)} \mathbb{1}_{(i, j) \in B_{r}} .
$$

(2) In the case of the contrast function $C_{2}$ with two balls of radius $r_{1}<r_{2}$,

$$
c_{i j}=\frac{1}{b\left(r_{1}\right)} \mathbb{1}_{(i, j) \in B_{r_{1}}}-\frac{1}{b\left(r_{2}\right)} \mathbb{1}_{(i, j) \in B_{r_{2}}},
$$

where $\mathbb{1}_{A}$ denotes the characteristic function of the event $A$ (its value is 1 is $A$ is true and it is 0 otherwise).

Therefore, the computations of the laws will be similar and they will be treated simultanously using the coefficients $c_{i j}$.

When the contrast function $C_{2}$ is used with two radii $r_{1}<r_{2}$, we compute the Laplacian $\Delta_{r} I$ with the larger ball radius, that is with $r=r_{2}$.

Lemma 3 For both contrast functions $C_{1}$ and $C_{2}$, the vector

$$
\left(C_{x}(u, v), C_{y}(u, v), \Delta_{r} I(u, v)\right)
$$

is a Gaussian vector with mean 0 and covariance matrix of the form:

$$
\left(\begin{array}{ccc}
\sigma_{x}^{2} & 0 & \sigma_{x, \Delta} \\
0 & \sigma_{y}^{2} & 0 \\
\sigma_{x, \Delta} & 0 & \sigma_{\Delta}^{2}
\end{array}\right)
$$

In particular, we have that $C_{y}$ is independent of $\left(C_{x}, \Delta_{r} I\right)$.

Proof: The lemma is a consequence of the two following remarks. The first one is that, in both cases for the contrast function, the coefficients $c_{i j}$ are 
symmetric: $c_{i, j}=c_{-i, j}$ and $c_{i, j}=c_{i,-j}$. Thus they satisfy that whenever $k$ or $l$ is odd then

$$
\sum_{(i, j) \in B_{r}} i^{k} j^{l} c_{i j}=0
$$

The second remark is that the vectors $\vec{I}_{i}$ and $\vec{I}_{k}$ are independent if $i \neq k$. And we thus have

$$
\mathbb{E}\left[I_{i, j} I_{k, l}\right]= \begin{cases}0 & \text { if } i \neq k, \\ \Gamma(j, l) & \text { if } i=k\end{cases}
$$

where $\mathbb{E}[X]$ denotes the expectation (i.e. the mean value) of the random variable $X$.

We can now compute the covariance matrix. For instance, let us start with:

$$
\begin{aligned}
\mathbb{E}\left[C_{x} C_{y}\right] & =\sum_{(i, j, k, l)} j k c_{i j} c_{k l} \mathbb{E}\left[I_{u+i, v+j} I_{u+k, v+l}\right] \\
& =\sum_{(i, j, l)} j i c_{i j} c_{i l} \Gamma(v+j, v+l) \\
& =\sum_{(j, l)} j \Gamma(v+j, v+l) \sum_{i} i c_{i j} c_{i l}=0 .
\end{aligned}
$$

Similar computations give $\mathbb{E}\left[C_{y} \Delta_{r} I\right]=0$ and

$$
\begin{aligned}
\sigma_{x}^{2} & :=\mathbb{E}\left[C_{x}^{2}\right]=\sum_{(i, j, l)} j l c_{i j} c_{i l} \Gamma(v+j, v+l) ; \\
\sigma_{y}^{2} & :=\mathbb{E}\left[C_{y}^{2}\right]=\sum_{(i, j, l)} i^{2} c_{i j} c_{i l} \Gamma(v+j, v+l) ; \\
\sigma_{\Delta}^{2} & :=\mathbb{E}\left[\left(\Delta_{r} I\right)^{2}\right]=\frac{1}{\beta^{2}(r)} \sum_{(i, j, l) \in \Omega_{r}}\left(\alpha(r)+i^{2}+j^{2}\right)\left(\alpha(r)+i^{2}+l^{2}\right) \Gamma(v+j, v+l) ; \\
\sigma_{x, \Delta} & :=\mathbb{E}\left[C_{x} \Delta_{r} I\right]=\frac{1}{\beta\left(r_{2}\right)} \sum_{(i, j, l) \in \Omega_{r_{2}}} j c_{i j}\left(\alpha(r)+i^{2}+l^{2}\right) \Gamma(v+j, v+l),
\end{aligned}
$$

where we have set $\Omega_{r}=\left\{(i, j, l)\right.$ such that $(i, j) \in B_{r}$ and $\left.(i, l) \in B_{r}\right\}$.

\subsection{Computation of the threshold}

As the first and the second order derivatives are no longer independent, we must compute the conditional law of the contrast function knowing that $\Delta_{r} I=$ 0 . 
Proposition 2 Let $C$ be one of the two contrast functions. Then, the random variable $\|C\|^{2}$ is distributed, conditionally on $\left\{\Delta_{r} I=0\right\}$, as the sum of the square of two independent Gaussian random variables, with mean zero and respective variance

$$
\sigma_{y}^{2} \quad \text { and } \quad \sigma_{x \mid \Delta=0}^{2}=\frac{\sigma_{x}^{2} \sigma_{\Delta}^{2}-\sigma_{x, \Delta}^{2}}{\sigma_{\Delta}^{2}}
$$

that is a Gamma law with parameters $\frac{1}{2}$ and $\frac{1}{2}\left(\sigma_{y}^{2}+\sigma_{x \mid \Delta=0}^{2}\right)$.

Remark: The threshold value $s(\varepsilon)$ defined by

$$
\mathbb{P}\left(\|C\| \geq s(\varepsilon) \mid \Delta_{r} I=0\right) \leq \varepsilon
$$

can no longer be computed explicitly but a numerical approximation is easy to get as the Gamma density is well-known.

Proof: $C_{y}$ is independent of the pair $\left(C_{x}, \Delta_{r} I\right)$. Thus, conditionally on $\left\{\Delta_{r} I=\right.$ $0\}$, the random variables $C_{y}$ and $C_{x}$ are still independent and the conditional law of $C_{y}$ is the Gaussian distribution with mean 0 and variance $\sigma_{y}^{2}$.

Now, if $D^{2}:=\sigma_{x}^{2} \sigma_{\Delta}^{2}-\sigma_{x, \Delta}^{2} \neq 0$, then the law of the pair $\left(C_{x}, \Delta_{r} I\right)$ has a density which is given by

$$
f_{x, \Delta}\left(t_{1}, t_{2}\right)=\frac{1}{2 \pi D} e^{-\frac{1}{2}\left(t_{1}, t_{2}\right) \Lambda\left(t_{1}, t_{2}\right)^{t}}
$$

where $\Lambda$ is the inverse of the covariance matrix, i.e.

$$
\Lambda=\frac{1}{D^{2}}\left(\begin{array}{cc}
\sigma_{\Delta}^{2} & -\sigma_{x, \Delta} \\
-\sigma_{x, \Delta} & \sigma_{x}^{2}
\end{array}\right)
$$

Let us recall that, if $f_{\Delta}$ denotes the Gaussian density of $\Delta_{r} I$, then the law of $C_{x}$ conditionally on $\Delta_{r} I=0$ has a density given by

$$
\frac{f_{x, \Delta}\left(t_{1}, 0\right)}{f_{\Delta}(0)}
$$

and so is Gaussian with mean zero and variance

$$
\sigma_{x \mid \Delta=0}^{2}=\frac{D^{2}}{\sigma_{\Delta}^{2}}
$$

This result is still valid when $D=0$ since it implies that $C_{x}$ and $\Delta_{r} I$ are proportional and thus the law of $C_{x}$ conditionally on $\Delta_{r} I=0$ is Gaussian with mean 0 and variance 0 (it is not random anymore). 


\subsection{Experiments}

\subsubsection{Case of a piecewise constant object}

To begin with, we still study the piecewise constant object of Figure 1 described in Section 3.4. Let us recall that this image represents a slice of the object that contains the symmetry axis. The 3 -dimensional object is obtained by rotation around the vertical axis that goes through the middle of the image.

In that case, we will use the contrast function $C_{1}$, which is simply the norm of the gradient. The experiments of Figure 9 correspond to a ball radius $r=12$ pixels. The size of the images is $512 \times 512$ pixels.

We start with the image of the radiograph obtained after the application of matrix $H$ to our initial image. Then a Gaussian white noise (with $\sigma=4$ ) is added to this radiograph. Then tomographic inversion (application of the matrix $\mathrm{H}^{-1}$ ) is performed. This gives the image of Figure 9(a). As we already mentioned it, the noise is not stationary, it is now correlated and its variance depends on the distance from the symmetry axis. For instance, if the standard deviation of the Gaussian white noise on the radiograph is $\sigma=4$, the variance of the noise on the tomography is about $2 \sigma^{2}=32$ near the axis, $0.02 \sigma^{2}=0.32$ at a distance of 65 pixels from the axis and $8.10^{-3} \sigma^{2}=0.128$ at the edge of the image located on the right at 200 pixels from the axis.

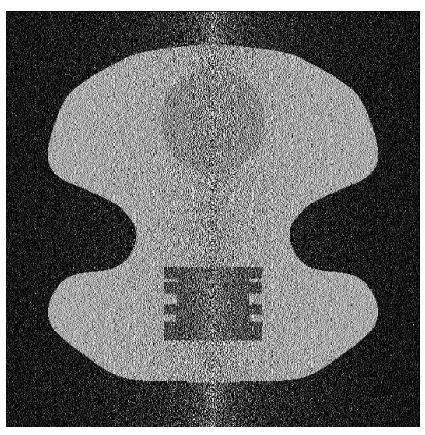

(a)

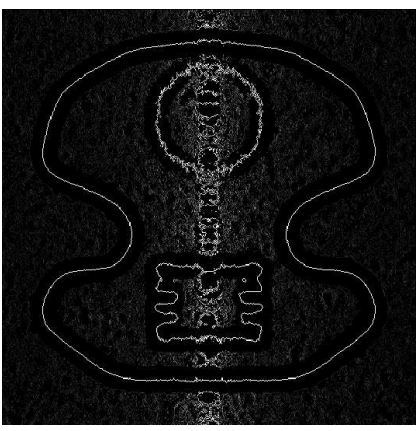

(b)

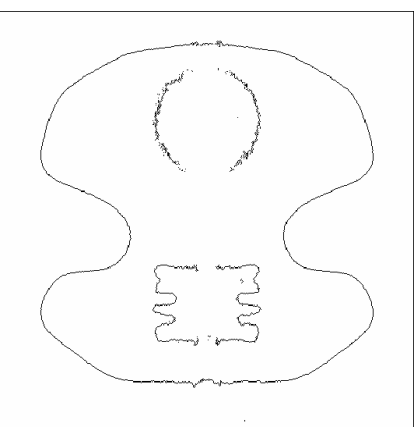

(c)

Fig. 9. (a) Reconstructed object from a single noisy radiograph, (b) Contrast value at the zero-crossings of the Laplacian for the contrast function $C_{1}$, (c) Significant edges.

The threshold value can be numerically computed. As an illustration, we display on Figure 10 the threshold value for the contrast $C_{1}$ as a function of the distance from the symmetry axis (range from -256 to +256 ), in the case of a noise level $\sigma=4$ and of a ball radius $r=12$.

Some edges are not declared significant by our algorithm near the symmetry axis; the noise is too pronounced there to distinguish the true edges from the 


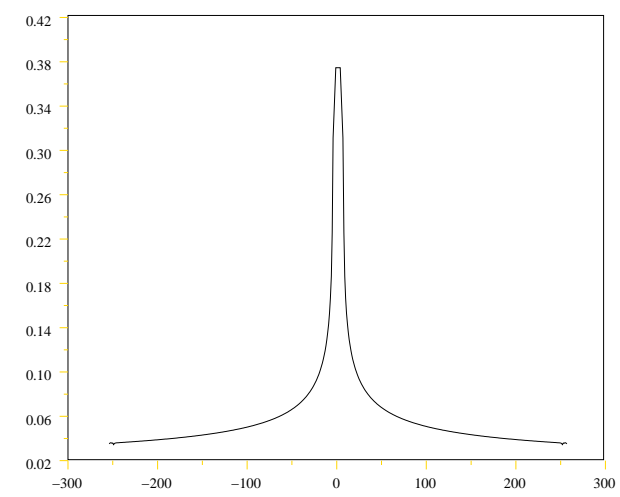

Fig. 10. Numerical computation of the threshold as a function of the distance from the symmetry axis for the noise level $\sigma=4$ in the case $r=12$.

noise. In other words, the threshold value near the symmetry axis (see Figure 10) can be higher than the contrast associated with small differences in the density of the object. In those images, an edge with a low contrast is significant if it is far enough from the symmetry axis (see also Figure 11). Even when the edges are significant, a high level of noise leads to a decrease in the location accuracy and increased raggedness of the edges. Moreover, some details are lost because of the smoothing due to the size of the ball.

Since the edges separate two materials, one included in another, they must be closed curves. Currently, a human operator has to close them manually. Our method yields open edges. This does not imply that there is no frontier between the materials but only that the noise level is too high to give an accurate position of the edge. Therefore, we can either close the curves manually, or with standard curve completion methods, but this will not tell which closure is better (i.e. the closest to the real shape). Several methods are usual for curve completion. Let us mention

- Methods based on graph exploration [7],

- Methods inspired by Neurobiology [9],

- Snakes with fixed tips [5].

Choice of the ball radius. Let us compare the results obtained with different ball radii (see Figure 11). When the ball radius is small, the edges are more accurate but some are not significant: the smoothing of the noise is not enough to get rid of it. On the contrary, when the radius is large, most of the edges are detected but small details are lost because of this smoothing. 

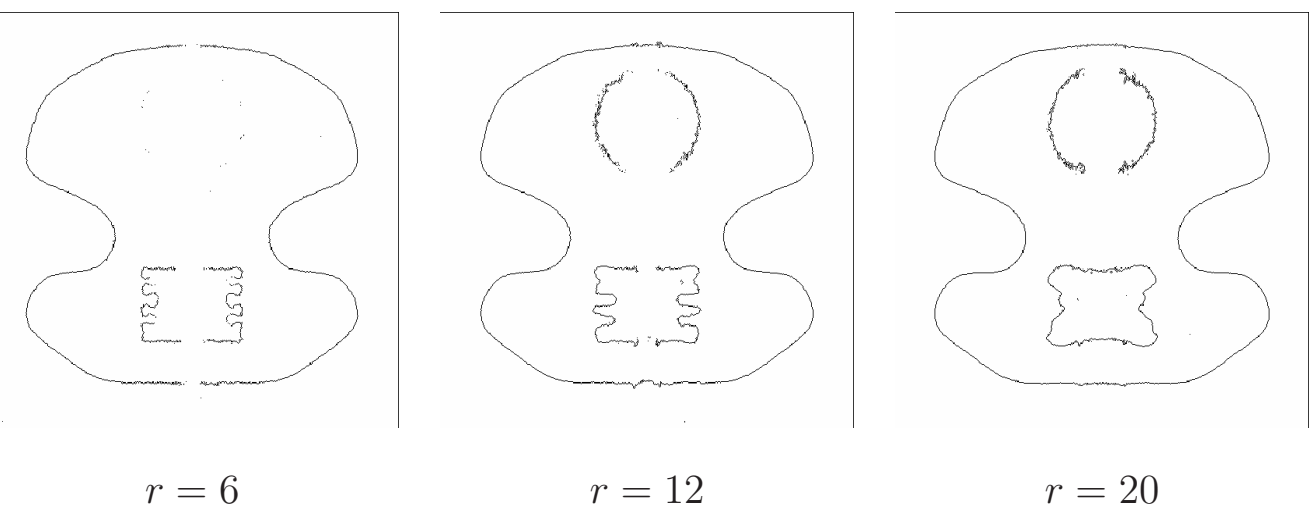

Fig. 11. Significant edges obtained with different ball radius: from left to right: $r=6, r=12$ and $r=20$

The choice of the ball radius depends essentially on the complexity of the edges (or the degree of accuracy we want). If we know (from prior knowledge) that the edges are very smooth, a large radius give very good results (see the outer edge of our studied object). On the contrary, if we look for very complicated objects, we must choose smaller radii but some edges (with low contrast) may become non-significant.

The choice of radius 12 corresponds to an appropriate balance between the size of typical details and an effective smoothing of the noise in our case.

Comparison with other methods. We will give here the results obtain with two other methods which have both the advantage of directly providing closed curves.

- The first method is the one introduced in [6]. One keeps only the meaningful level lines of the image, which are defined by: the minimum of the norm of the gradient along the level line is larger than a threshold $T(\varepsilon)$. This threshold is computed from the gradient histogram of the image. The meaning of this definition is that such curves have a probability less than $\varepsilon$ to appear in a pure noise image (with same gradient histogram as the original image). The results obtained with this method are shown on Figure 12. On the first row: we smooth the image of Figure 9(a) by convolution with a Gaussian kernel with respective standard deviation 2 and 4 pixels. And then, on the second row, we display the respective meaningful level lines. This experiment clearly shows that, since the noise model is not adapted to the image (in particular, the non-stationarity is not taken into account), many false contours are detected. - The second method is the famous Mumford-Shah segmentation for piecewise constant images [15]. Given an observed image $g_{0}$ defined on a domain $D$, one looks for the piecewise constant approximation $g$ of $g_{0}$ that minimizes the functional

$$
E_{\lambda}(g)=\int_{D}\left|g-g_{0}\right|^{2}+\lambda \operatorname{Length}(K(g)),
$$

where Length $(K(g))$ is the one-dimensional measure of the discontinuity set 

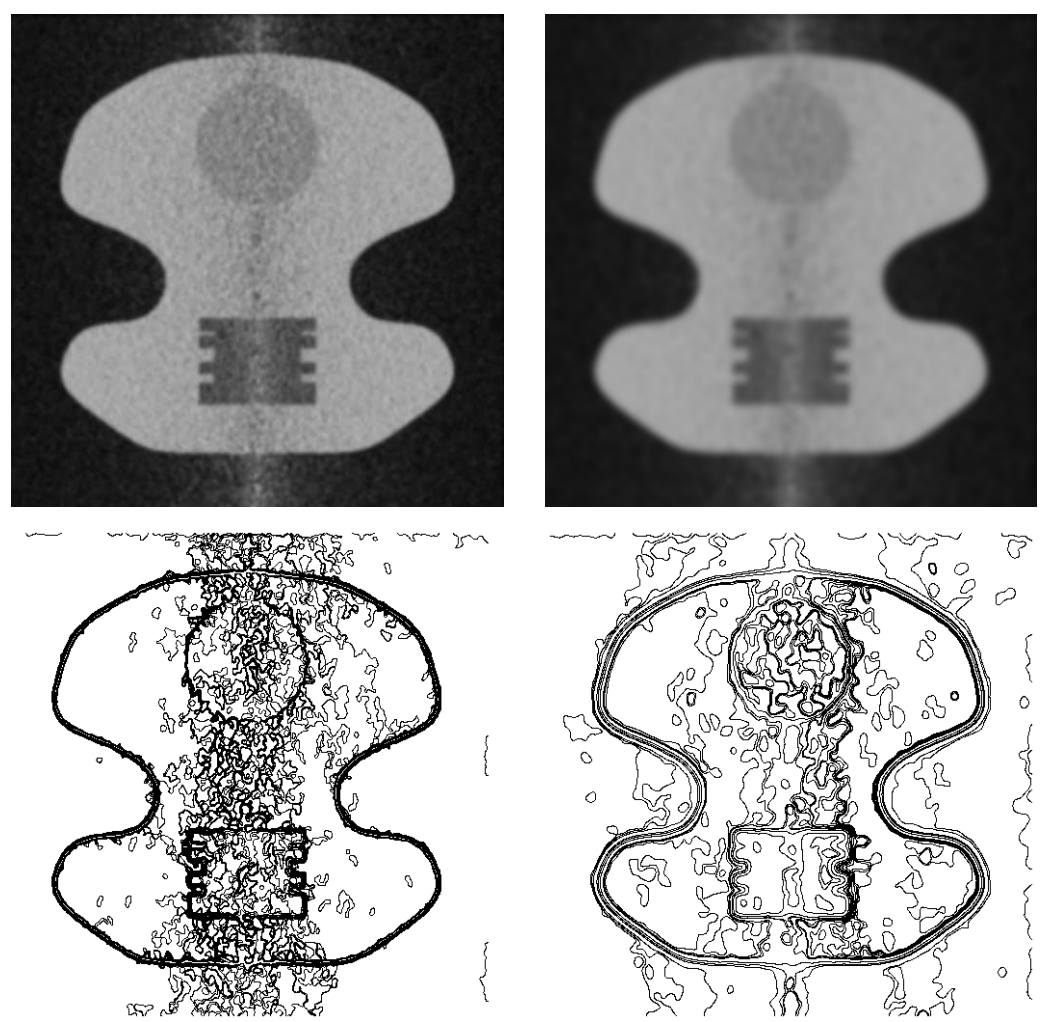

Fig. 12. First row: the image of Figure 9(a) is smoothed by convolution with a Gaussian kernel with respective standard deviation 2 and 4 pixels. Second row: the meaningul level lines of each image.
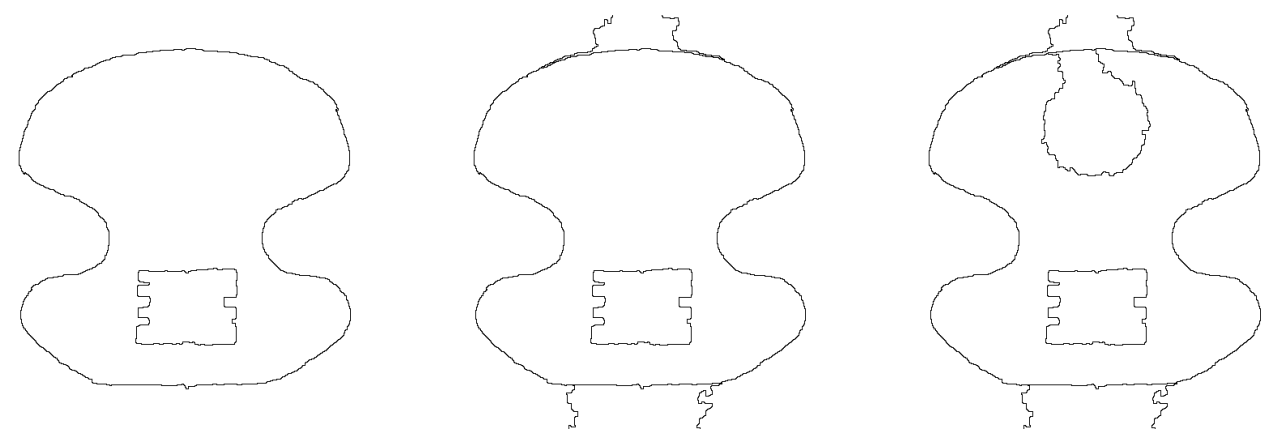

Fig. 13. Results obtained with the Mumford-Shah segmentation for piecewise constant images, for three different values of $\lambda$ (see text). From left to right, the number of regions in the segmented image is respectively 3,6 and 7 .

of $g$ (which is a set of curves denoted by $K(g)$ ) and $\lambda$ is a parameter which weights the second term of the functional. The results obtained with this method are shown on Figure 13 for three different values of $\lambda$ : respectively $\lambda=42882, \lambda=12220$ and $\lambda=11621$ (the size of the image is $512 \times 512$ ). The implementation used here is the region-merging algorithm described in [12]. The main drawback of this method is that there is no verification that the obtained contours are not due to the noise, and moreover the user has to give the value of $\lambda$ (or of the final number of regions). 

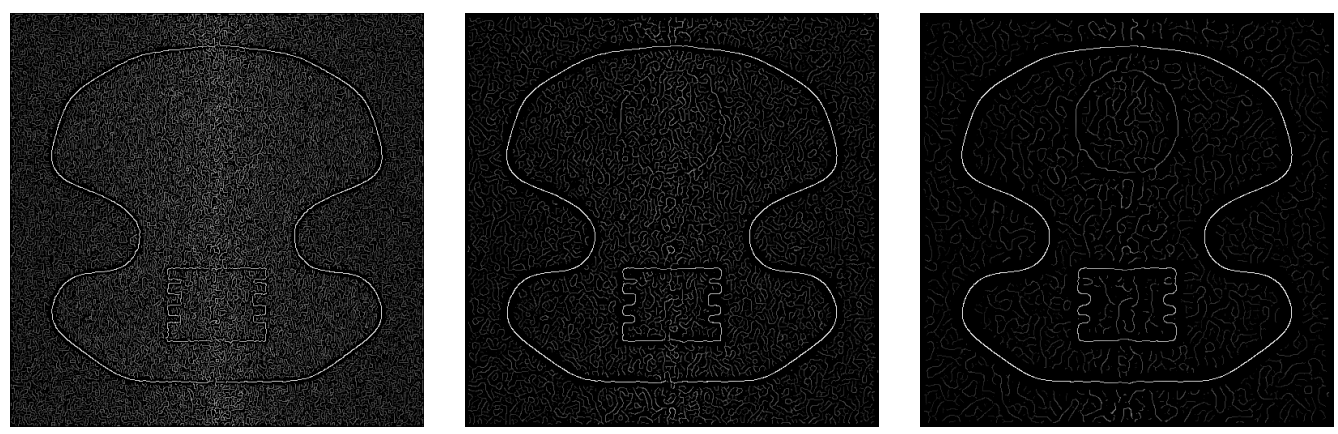

Fig. 14. From left to right: results of the Canny edge detector for respectively, the original image (Figure 9(a)), the image smoothed by convolution with a gaussian kernel of width $\sigma=2$ (middle) and $\sigma=4$ (see first row of Figure 12).

We end this subsection with the results of Canny detector on the original image and on the images obtained after Gaussian filtering with respective standard deviation $\sigma=2$ and $\sigma=4$. The results are shown on Figure 14. On these edge maps, no thresholding has been performed. The next step would be to compute the significant edge points. According to the definition of a significant edge point, the question would be: $I$ being a pure noise image (with known parameters), what is the law of $|\nabla I|(x)$ conditionally on $x$ being a Canny edge point ? Such a computation is difficult to perform and should be part of some future work. The obtained result would probably be close to the ones obtained with our method (see for instance Figure 11).

\subsubsection{Case of an inhomogeneous material}

Let us turn now to a more realistic case: the materials are not homogeneous and consequently the object is no longer piecewise constant (see Figure 15(a)). The object is now composed of an inhomogeneous material (with constant gradient) with homogeneous objects inside. In the first experiment, the noise level is $\sigma=4$. The results (Figures 15 and 16) are similar to the ones obtained for an homogeneous object in the previous section.

In the second experiment (Figure 17), the noise level is $\sigma=0.4$. It is lower than the one used in the previous experiments, and this is why the artifacts of tomographic reconstruction are less pronounced here. The noise level here is such that the threshold computed with the contrast function $C_{1}$ is lower than the gradient of the material. In that case the use of the contrast function $C_{1}$ fails as many detected edges remain significant. This is illustrated by Figure 18(a). Figure 18(b) gives the significant edges obtained with the contrast function $C_{2}$ with two ball radii $r_{1}=6$ and $r_{2}=12$. With this contrast function, we eventually get only the "true" edges.

Choice of the ball radii. Here again, we must discuss how the radii are chosen. The discussion concerning the choice of the radius of the outer ball is exactly 


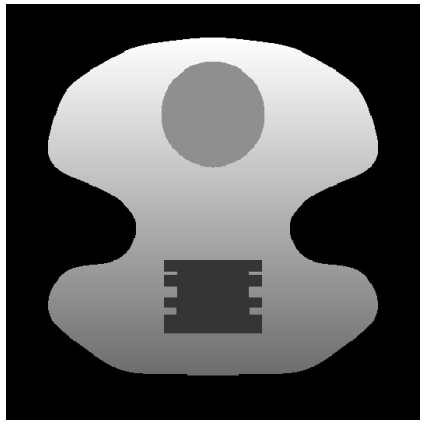

(a)

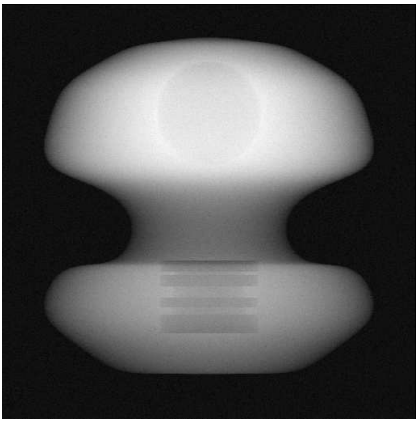

(b)

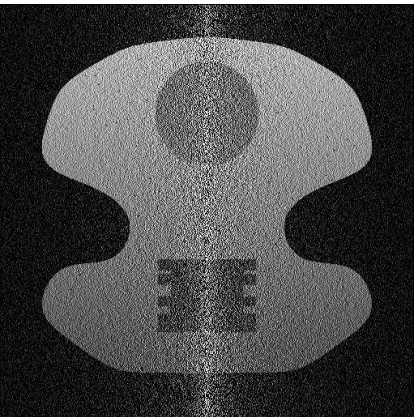

(c)

Fig. 15. (a) Inhomogeneous object, (b) Its noisy radiograph $(\sigma=4)$, (c) Tomographic reconstruction.

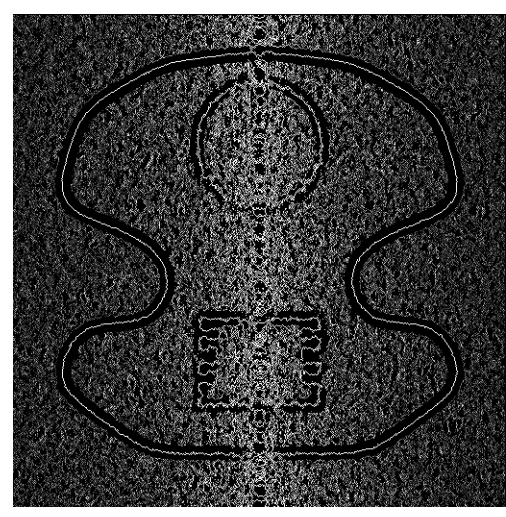

(a)

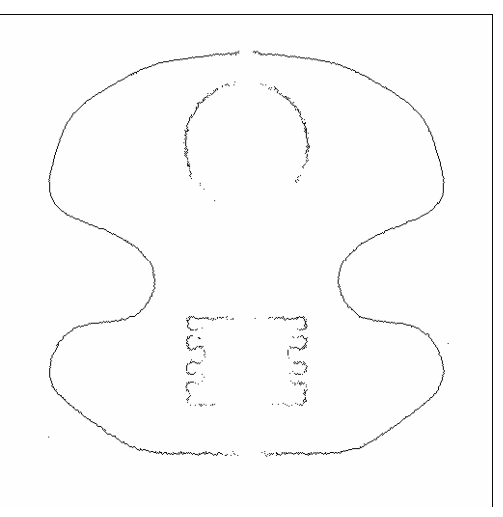

(b)

Fig. 16. (a) Contrast value at the zero-crossings of the Laplacian for the contrast function $C_{1}(r=12)$, (b) Significant edges of the inhomogeneous object.

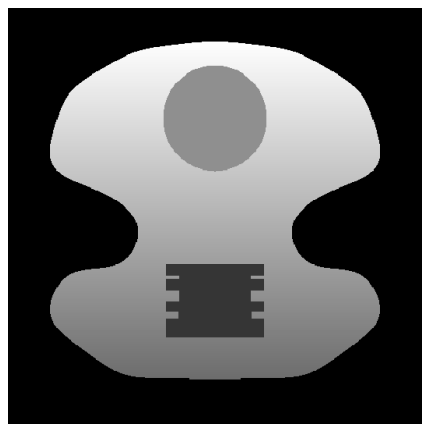

(a)

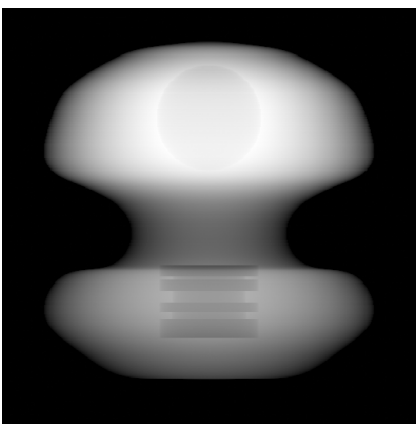

(b)

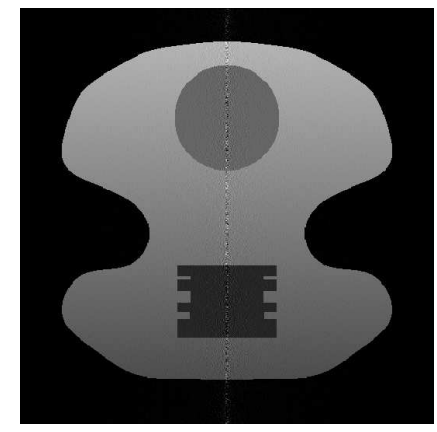

(c)

Fig. 17. (a) Inhomogeneous object, (b) Its noisy radiograph $(\sigma=0.4)$, (c) Tomographic reconstruction

the same as for the contrast function $C_{1}$. That is why we kept the value of 12 . The inner ball radius must not be too close to the outer one as the difference between the two gradients would be too small. But this radius must also be not too small in order to still perform a good smoothing of the noise. The 


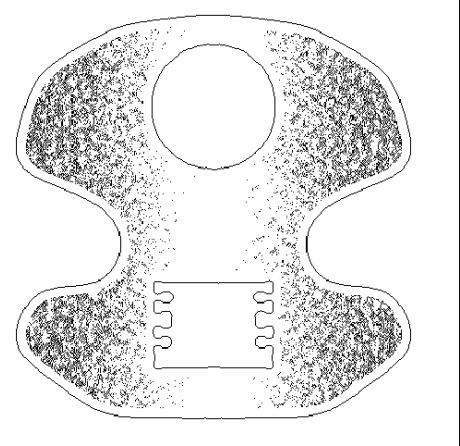

(a)

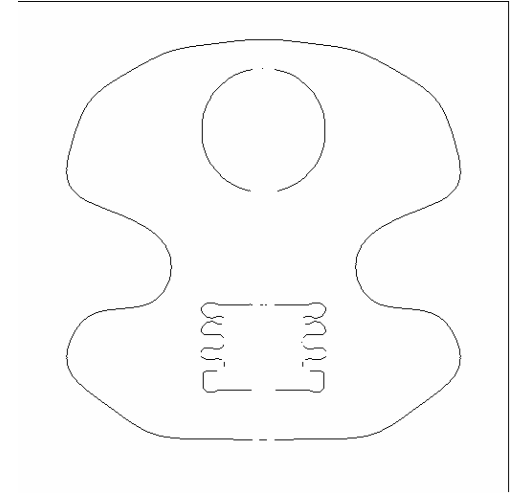

(b)

Fig. 18. (a) Significant edges with the $C_{1}$ contrast function $(r=12)$ : there are many false detections. (b) Significant edges with the $C_{2}$ contrast function $\left(r_{1}=6\right.$ and $r_{2}=12$ ): only the "true" edges are obtained.

choice of the half of the outer radius is usually a good compromise.

\section{Discussion and conclusion}

In this paper, we have introduced a method for the detection of significant edges in noisy images when the parameters of the noise are known. The general idea of the method is to make a statistical test to decide whether an edge point is due to the noise or not.

The edge detector we have used here is the zero-crossings of an estimated Laplacian of the image. We did not use a Gaussian filter to smooth the image and estimate its Laplacian (as it is done in Marr-Hildreth detector [14]), but rather local polynomial approximations of the image. The reason for this is that such an estimator is more robust to noise.

We think that future extensions of our work should mainly focus on:

- performing the same kind of computations for Marr-Hildreth detector, for Canny detector, and more generally for "derivative-based" edge detector (including non-isotropic ones);

- being able to merge the informations obtained at different scales (which are the different ball radii in our case). Indeed, as we have shown it in the experimental section, it is sometimes necessary to use the gradient estimated at two different scales to obtain good results;

- extending the framework to tomography without rotational symmetry, and more generally to other situations of non-stationary noise (including possibly non-Gaussian ones). 


\section{References}

[1] I. Abraham, R. Abraham, J.-M. Lagrange and F. Lavallou, Méthodes inverses pour la reconstruction tomographique X monovue, Revue Chocs 31 (chocs@cea.fr), 2005.

[2] R.N. Bracewell, The Fourier transform and its applications, 2nd edition, Mc Graw-Hill New-York, 1978.

[3] J. Canny, A computational approach to edge detection, IEEE Trans. on Pattern Analysis and Machine Intelligence 8, pp. 679-698, 1986.

[4] F. Cao, P. Musé and F. Sur, Extracting Meaningful Curves from Images, Journal of Mathematical Imaging and Vision 22, pp. 159-181, 2005.

[5] L.D. Cohen, Etudes des modèles de contours actifs et autres techniques de traitement d'image, PHD Thesis, University Paris-Sud, 1990.

[6] A. Desolneux, L. Moisan and J.-M. Morel, Edge Detection by Helmholtz Principle, Journal of Mathematical Imaging and Vision 14, pp. 271-284, 2001.

[7] R. Deriche, J.P. Cocquerez, G. Almouzni. An efficient method to build early image description. 9th Int. Conference on Pattern Recognition, Roma, 1988.

[8] J.M. Dinten, Tomographie à partir d'un nombre limité de projections : Régularisation par des champs markoviens, PhD Thesis, Université Paris-Sud, 1990.

[9] P. Gaussier, J.P. Cocquerez, Utilisation des réseaux de neurones pour la reconnaissance de scènes complexes : simulation d'un système comprenant plusieurs aires corticales, Traitement du signal 6, pp. 441-466, 1991.

[10] T. Hida, Brownian Motion, Applications of Mathematics 11, Springer-Verlag, 1980.

[11] T. Hida, H.H. Kuo, J. Potthoff and L. Streit, White Noise. An infinite Dimensional Calculus. Mathematics and its Applications 253, Kluwer Academic Publishers Group, Dordrecht, 1993.

[12] G. Koepfler, C. Lopez and J.-M. Morel, A Multiscale Algorithm for Image Segmentation by Variational Method, SIAM Journal on Numerical Analysis 31 (1), pp. 282-299, 1994.

[13] D. Marimont and Y. Rubner, A probabilistic framework for edge detection and scale selection, 6th Int. Conference on Computer Vision, 1998.

[14] D. Marr and E. Hildreth, Theory of edge detection, Proceedings of the Royal Society of London B 207, pp. 187-217, 1980.

[15] D. Mumford and J. Shah, Boundary detection by minimizing functionals, Proc. IEEE Conference on Computer Vision and Pattern Recognition, San Francisco, 1985. 
[16] P. Qiu and S. Bhandarkar, An edge detection technique using local smoothing and statistical hypothesis testing, Pattern Recognition Letters 17, pp. 849-872, 1996.

[17] M. Sezgin and B. Sankur, Survey over image thresholding techniques and quantitative performance evaluation, Journal of Electronic Imaging 13(1), pp. 146-165, 2004.

[18] R. Touzi, A. Lopes and P. Bousquet, A statistical and geometrical edge detector for SAR images, IEEE Transactions on Geoscience and Remote Sensing 26, pp. 764-773, 1988.

[19] O.D. Trier and T. Taxt, Evaluation of Binarization Methods for Document Images, IEEE Transactions on Pattern Analysis and Machine Intelligence 17 (3), pp. 312-315, 1995.

[20] J.B. Walsh, An introduction to stochastic partial differential equations, Ecole d'été de Probabilités de Saint-Flour XIV 1984, Lecture Notes in Math. 1180, Springer, Berlin, 1986. 